\title{
Physical organic chemistry in the making
}

\author{
Jan B. F. N. Engberts \\ Department of Organic and Molecular Inorganic Chemistry, University of Groningen, Nijenborgh \\ 4, 9747 AG Groningen, The Netherlands,
}

Biography: Jan B. F. N. Engberts was born in Leiden, The Netherlands and grew up in Amsterdam. $\mathrm{He}$ received his undergraduate and graduate education at the University of Groningen. After his $\mathrm{Ph}$. D. (with honors) in 1967, he was a postdoc at the University of Amsterdam, doing free-radical chemistry. After returning to Groningen, he became Professor of General Chemistry at the same University in 1978. He is currently Professor of Physical Organic Chemistry, and his research is focussed on reactivity, molecular assembly, and molecular recognition processes in water. In these and related areas ca. 325 papers have been published. He was visiting professor at the Universities of Leuven (Belgium) and Bratislava (Slovakia). His hobbies include process philosophy, horseback riding, and poetry.

\begin{abstract}
The discipline of physical organic chemistry will continue to occupy a central position in chemistry. The rapid increase in instrumentation and important theoretical developments allow the investigation of many problems of great complexity and challenge. In the next century the leading theme will continue to be the quantitative analysis of the effects of structural variation on the properties of molecules and molecular assemblies.
\end{abstract}

Physical organic chemistry originated at the time that the advancement of theory and the development of experimental facilities allowed the evolution of quantitative thinking in the design, synthesis, and study of organic compounds. The early and exciting phase, roughly starting in the late thirties and early forties, culminated in the publication of influential textbooks, written by the leading pioneers in the field (1). Most emphasis was placed on reaction mechanisms in solution and the development of reliable criteria to pin them down. Important issues were kinetic analysis and the interpretation of substituent and isotope effects. Thermodynamic data often gave a strong starting point from which interpretations, sometimes quite speculative, at the molecular level were extracted (2). The possible consequences of extrathermodynamic assumptions were, however, in many cases recognized. Quantum chemical approaches slowly diffused into physical organic chemistry and eventually led to impressive insights into the electronic structure of organic compounds $(3,4)$. A classical and highly useful approach for analyzing the effects of structural variation was couched in terms of linear free energy relationships (LFER) (5). Those theories are still popular because of the unsurmountable problems in $a b$ initio calculations of rate constants and other relevant kinetic and thermodynamic parameters. In later years, rapid development of fast computing facilities and the burst in the availability of spectroscopic methods of ever increasing sensitivity (particularly NMR) allowed chemists to tackle problems of increasing complexity and sophistication. The disciplines of physical organic chemistry and (classical) physical chemistry came closer to each other and interests emerged in fields rather different from reaction mechanisms per se. For example, physical organic chemistry showed its direct relevance for understanding biochemical problems, including questions centered around the high efficiency of enzyme catalysis. In this respect, the monograph written by W. P. Jencks was a landmark (6). Influential novel developments also include host-guest chemistry (often referred to as supramolecular chemistry, three Nobel laureates in 1989) (7) and membrane-mimetic chemistry, relevant for understanding cell function $(8,9)$. The latter fields of research signal the shift in interest from covalent to non-covalent interactions, and the biochemical and industrial relevance of those fields is beyond doubt. Still more recently, molecular modeling became a popular approach, but these activities called for a critical appraisal of the force fields to avoid insignificant results and overinterpretation. Monte-Carlo and molecular dynamics simulations also exhibited their potential for solving physical organic problems. These computational approaches will in the future become more strongly incorporated into physical organic chemistry (10). 
All these recent developments inevitably led to vaguer notions about what exactly should be defined as physical organic chemistry. Is it a less rigorous branch of physical chemistry? Or is it a tool which could be selected for solving particular issues in material science, in the biosciences, and in polymer chemistry? The situation may be illustrated by a closer look at the research projects presently embedded in the Working Party on Physical Organic Chemistry, which is a section of the Netherlands Foundation for Chemical Research (SON). These topics include NMR in molecular biology (Prof. R. Kaptein), bioorganic chemistry of glycoconjugates and carbohydrates (Prof. J. F. G. Vliegenthart), chemistry of ions in the gas phase (Prof. N. M. M. Nibbering), photoinduced electron transfer in multichromophoric systems (Prof. J. W. Verhoeven), and design and study of (supra)molecular and macromolecular systems (Prof. R. J. M. Nolte). None of the twenty-four participating groups is fully focussed on the study of reaction mechanisms. The broadness of the field is perhaps also reflected in the absence of a substantial number of recent and modern textbooks that claim to cover comprehensively the essentials of the discipline. Nevertheless, the field is healthy and within university educational programs, reaction mechanisms are now incorporated into general organic chemistry courses. Furthermore, general organic chemistry textbooks based upon a mechanistic approach are popular (11). The prominent position of physical organic chemistry has been adequately described in a recent book on the history of chemistry (12). Last but not least, jobs continue to be available for young physical organic chemists in the chemical industry, where their broad scientific background is being recognized, as well as their ability to interact satisfactorily with engineers. It is clear that physical organic chemistry is rapidly expanding towards new horizons, not previously associated with the discipline. In this respect, it should be emphasized that physical organic chemistry has always been problem-driven rather than technique-driven. Some chemists develop a particular technique and then have a solution looking for a problem. By contrast, physical organic chemists have used a whole range of techniques, using the most appropriate one in each case. Often the problems chosen by physical organic chemists have been at the leading edge of current chemistry.

I expect that in the next decades the challenge for physical organic chemists will be found in a number of often multidisciplinary areas such as supramolecular chemistry, nanotechnology, surfactant and membranemimetic chemistry, catalytic processes, reactions inspired by biological transformations, non-equilibrium processes, and organic materials with specialized (super)conducting, optical, and magnetic properties. In a recent thought-provoking book, Philip Ball makes a number of suggestions (13) regarding the future possibilities for "designing the molecular world". In these activities, which ask for creativity and imagination, physical organic chemists have much to offer. In a happy marriage of sophisticated synthesis and physicochemical measurements, fields can be explored in which molecules are produced which are able to carry out specific functions. Examples include carrier systems for piloting drugs to the cell nucleus, highly specific and efficient catalysts, molecules that recognize each other with high specificity (particularly in water, the "matrix of life"), and molecules with desirable properties from the viewpoint of environmentally safe industrial applications. Interest will, of course, continue in classical issues like detailed reaction mechanisms, solvent effects, and (ultra)fast reactions (electron transfer, tunneling). In view of the broadening of the research activities of physical organic chemists, some scientists think that it is a legitimate question whether or not the field remains recognizable and worthy of being called a major chemical discipline.

I contend that in the future physical organic chemistry will continue to be largely characterized by detailed and quantitative analyses of the effects of structure variation on the properties of molecules and ensembles of molecules. The physical organic chemist will further contribute to developing a sophisticated feeling for what structural perturbation can teach us and will be able to synthesize the compounds necessary for such an approach. I think it is this expertise that clearly distinguishes the physical organic chemist from the physical chemist. New compounds will become available for probing structure-property relationships and the results will challenge theoretical chemists to employ statistical thermodynamic techniques to model these novel systems. Organic molecules respond to structure variation by exhibiting modifications in reactivity, in spectroscopic properties, in thermodynamic properties, and in many other respects. By identifying structureproperty relationships physical organic chemists provide, inter alia, ground rules for specialist synthetic organic chemists in the context of target-synthesis in, for example, the pharmaceutical sciences. In addition, they will provide a major contribution to establishing a firm basis for understanding the relation between molecular structure and physiological action. This subject will become increasingly important in biochemistry and in the medical sciences.

The physical organic chemist will be the scientist able to take advantage of the effects of structure pertur- 
bation for developing deeper insights into chemical behavior and for designing and understanding the molecular world as demanded by civilized societies.

\section{ACKNOWLEDGEMENT}

Stimulating discussions with Prof. M. J. Blandamer, Prof. A. J. Kirby, Prof. R. J. M. Nolte, and Prof. J. W. Verhoeven are gratefully acknowledged.

\section{REFERENCES}

1. A selection includes: (a) L. P. Hammett, Physical Organic Chemistry, McGraw-Hill, New York (1940); (b) C. K. Ingold, Structure and Mechanism in Organic Chemistry, Cornell Univ. Press, Ithaca (1953); (c) J. H in e, Physical Organic Chemistry, McGraw-Hill, New York (1956); (d) E. S. Gould, Mechanism and Structure in Organic Chemistry, Holt, Rinehart and Winston, New York (1959).

2. J. E. Leffler and E. Grunwald, Rates and Equilibria of Organic Reactions, Wiley, New York (1963).

3. K. B. Wiberg, Physical Organic Chemistry, Wiley, New York (1964).

4. C. D. Ritchie, Physical Organic Chemistry, Dekker, New York (1990).

5. N. B. Chapman and J. Shorter, Eds., Advances in Linear Free Energy Relationships, Plenum Press, New Y o r k (1972).

6. W. P. Jencks, Catalysis in Chemistry and Enzymology, McGraw-Hill, New York (1969).

7. B. Dietrich, P. Viout and J.-M. Lehn, Macrocyclic Chemistry, VCH, Weinheim (1993).

8. T. Kunitake. Angew. Chem., Int. Ed. Engl. 31, 709 (1992).

9. J. H. Fendler, Membrane Mimetic Chemistry, Wiley-Interscience, New York (1982).

10. W. F. van Gunsteren and H. J. C. Berendsen, Angew. Chem., Int. Ed. Engl. 29, 992 (1990).

11. See, for example, S. H. Pine, Organic Chemistry, 5th. ed., McGraw-Hill, New York (1987).

12. W. H. Brock, The Fontana History of Chemistry, Fontana Press, London (1992).

13. P. Ball, Designing the Molecular World, Princeton Univ. Press (1994). 